\title{
Podoconiosis, the Non-Filarial Elephantiasis, a Means of Discrimination and Poverty in Ethiopia
}

\author{
Fikresilasie Samuel Tasew \\ Department of Medicine, Ambo University, Ambo, Ethiopia
}

Email address:

fikre16sam@gmail.com

To cite this article:

Fikresilasie Samuel Tasew. Podoconiosis, the Non-Filarial Elephantiasis, a Means of Discrimination and Poverty in Ethiopia. American Journal of Biomedical and Life Sciences. Vol. 3, No. 3, 2015, pp. 67-70. doi: 10.11648/j.ajbls.20150303.17

\begin{abstract}
Podoconiosis is a non-infectious geo-chemical disease caused by exposure of bare feet to red clay soil derived from volcanic rocks. In Ethiopia, 1 million people suffer with the disease. It affects the physical condition (disability and deformity) of patients and also the social and economic status of affected individuals. Studies conducted in endemic countries clearly stated that there is stigmatization in the family and in general in the community. This consequences mental distress in affected individual. Besides, individuals affected economically: they cost for treatment and mainly they loss their productivity potential. These results financial crisis individually and aggravate poverty in the society. This review intends to address the social exclusion and economic impact of podoconiosis in Ethiopia.
\end{abstract}

Keywords: Podoconiosis, Discrimination, Poverty, Ethiopia

\section{Introduction}

Podoconiosis is endemic non-filarial elephantiasis of the lower legs and is thought to be caused by prolonged exposure of the feet to red clay soils of volcanic origin. This prolonged exposure when matched with genetic susceptibility to the disease results in damage to the lymphatic vessels and swelling of the lower legs over time (Davey, Gebrehannaet al 2007; TekolaAyele, Adeyemoet al. 2012).

It is found in at least 10 countries in tropical Africa, Central America and North West India, where such soils coexist with high altitude, high seasonal rainfall and low income. Podoconiosis develops in men and women working barefoot on irritant soils, with signs becoming apparent in most patients by the third decade of life. Colloid-sized silicate particles appear to enter through the skin, are taken up into macrophages in the lower limb lymphatics and cause endolymphangitis and obliteration of the lymphatic lumen (Davey et al. 2007)

According to Deribe et al. (2013b) report, the prevalence of podoconiosis in Ethiopia ranges from $0.0 \%$ to $48.0 \%$. The high prevalence of podoconiosis is occurred in the southwest and southern parts of the country. The highest prevalence was recorded in KelemWelega and Illubabor zones of Oromia. The prevalence of podoconiosis was generally lower in the far western part of the country, such as Gambella.
Podoconiosis causes substantial disability and deformity among those affected and can lead to psychological, social and physical consequences. It is often highly stigmatising for individuals and promotes impoverishment in the society (Mousley et al. 2015).More recent research conducted in Ethiopia has also described that, podoconiosis costs a Zone of 1.5 million inhabitants almost $\$ 16 \mathrm{~m}$ per a year as a result of lost productivity and medical costs. Besides, more than half the populations in podoconiosis-endemic areas harbor stigmatizing attitudes to patients (Davey, 2008). Hence, this review aimed to assess the status of discrimination in podoconiosis patient and its financial related crisis in affected individuals.

\section{Review}

Podoconiosis is an important yet neglected problem of social, economic, development and public health relevance in endemic countries. The disease has recently gained global and national attention. It has recently been recognized by the World Health Organization (WHO) as a neglected tropical disease (NTD) because of the disabling impact it has on affected communities (WHO, 2011). 


\section{Epidemiology and Risk Factors}

Globally, it is estimated that there are at least four million people with podoconiosis. The disease has been reported in more than 20 countries, of which ten had high burden of the disease. In endemic highland areas of these countries podoconiosis is more prevalent than commonly known diseases such as HIV/AIDS, tuberculosis, malaria, or filarial elephantiasis (Davey et al. 2007).

It is estimated that up to 1 million cases of podoconiosis (i.e. $25 \%$ of the global total case load) exist in Ethiopia. The 'at-risk' population for podoconiosis is made up of all the people who live and farm on irritant soil.The soil is estimated to cover $18 \%$ of the surface area of Ethiopia, on which estimated $22-25 \%$ of Ethiopia's population (19.3 million) lives. In endemic areas of Ethiopia, the prevalence of podoconiosis is high: $9.1 \%$ in Illubabor Zone, Oromia Region; 6\% in the Pawe resettlement area, northwest Ethiopia; $5.5 \%$ in Wolayta zone, SNNPR; $2.8 \%$ in GullisoWoreda, West Wollega zone, Oromia region, $7.4 \%$ in Midakegni, West Shewa Oromia region, 3.3\% in Debreelias and Dembecha, East and West Gojjam, Amhara region (FMOH, 2013 and Tekola et al. 2006)

Its distribution is limited under certain environmental conditions, presumably those conditions favorable for the weathering of rock to produce specific types of soil. Climatic conditions, primarily altitude, rainfall, precipitation and temperature, influence the weathering of rocks and determine the type of soil generated, which in turn probably influences the distribution of podoconiosis. High prevalence areas $(>5 \%)$ are characterized by mean altitude $>1500$ masl, temperature between $19-21^{\circ} \mathrm{C}$, mean annual rainfall $>1500$ $\mathrm{mm}$ and mean annual precipitation $>130 \mathrm{~mm}$. There may be other variables influencing prevalence which might need further analyses and work (Deribe, et al. 2013a)

Epidemiological and ecological studies conducted in soil environments of endemic areas have identified that phyllosilicate minerals, particularly clay (smectite and kaolinite) and mica groups, quartz (crystalline silica), iron oxide, and zirconium are strongly associated with podoconiosis prevalence. Presence of more quantities of smectite, mica and quartz within the soil highly affect the podoconiosis prevalence. These minerals may influence water absorption, potentiate infection and be toxic to human cells (Molla et al. 2014).

\section{Pathology}

Podoconiosis is characterized by a prodromal phase before elephantiasis sets in. Early symptoms commonly include itching of the skin of the forefoot and a burning sensation in the foot and lower leg. Early changes that may be observed are splaying of the forefoot, plantar oedema with lymph ooze, increased skin markings, hyperkeratosis with the formation of moss-like papillomata (left) and rigid toes. Later, the swelling may be one of two types: soft and fluid, or hard and fibrotic, often associated with multiple hard skin nodules.
Acute adenolymphangitis episodes occur in which the patient becomes pyrexial and the limb warm and painful. These episodes appear to be related to progression to the hard, fibrotic leg (WHO, 2011).

\section{Economic Impacts}

Besides the high distribution of Podoconiosis in endemic areas, which also set a huge impact on physical, social and economic burden of affected communities. Thus, patients cost for treatment, loose their productivity potential (wage earning potential) and have low quality of life in the community than non-affected individuals.

Studies have assessed the quality of life (QoL) of patients with podoconiosis in comparison with healthy controls in Ethiopia. The report indicated that patients with podoconiosis have significantly lower QoL than healthy controls, both in the overall score and all domains of quality of life (physical, psychological, social and environmental). It has also shown that patients with podoconiosis have lower mean QoL scores than those with other NTDs such as lymphatic filariasis and leprosy (Mousley et al. 2013)

Podoconiosis has enormous economic impact in affected areas. Patients may cost for treatment purpose exhaustively. Some studies suggested that the total direct cost of podoconiosis is amounted to the equivalent of US\$ 143 per patient per a year. The productivity of the individual also decline significantly. Patients loss their productivity amounted to $45 \%$ of the total working days per year, which is causing a monetary loss equivalent to US\$ 63. In Wolaita zone, the overall cost of podoconiosis exceeds US\$16million per year. (Tekola et al. 2006).

The socioeconomic impact of the podoconiosis disease in Ethiopia is very high. In one study, out of 10 patients, seven to nine affected individuals are tend to belong to the economically active age group population, and it is estimated to result in a loss of USD 1.6 million per year in one of Ethiopia zone of 1.6 million people alone, this implying that at national level, the economic losses due to podoconiosis may be higher than USD 200 million per year (Tekola et al. 2006).

\section{Social Stigma}

Social stigma related to podoconiosis has a major impact on the psycho-social well being of patients. In endemic areas podoconiosis is one of the commonest causes of stigmatization against community members. The disease leads to social exclusion of individuals and their families.

In one study, the mean enacted stigma score was higher in 'major life areas', and 'community, social and civic life' than 'interpersonal interactions', while felt stigma was experienced most at the interpersonal level. In the studies related to stigmatization, patients commonly reported that they had considered suicide in response to discrimination and prejudice, particularly in interpersonal interactions. Forced divorce, dissolution of marriage plan, insults and exclusion at 
social events were some of the most commonly mentioned forms of enacted stigma reported patients (Tora et al.2014).The most Pronounced social stigma in endemic areas are often unable to marry, excluded from school, church, and social events (Tekola et al. 2006).

Podoconiosis patients frequently experience stigma in their day to day interactions with family members. Major causes of family stigma include unwillingness to cover care and treatment, a perception that the disease is contagious, fear of public identification of familial disease and inability to be economically productive (Tora et al. 2011).

Prejudice and discrimination against patients by family members exposes patients to deprivation of emotional and material support. As a result, the capacity to challenge family stigma is weak. Thus, most podoconiosis patients employ avoidant coping strategies and changing of relational meaning (Tora et al. 2011).

These stigmas, deformity and disability problems related to most neglected tropical diseases finally may lead to poor mental health in affected individuals. As a result, prevalence of mental distress is increased in podoconiosis affected individuals than healthy individuals, with a significant association of podoconiosis and mental distress (Mousley et al. 2015). The main reasons for prevailing such discrimination against patients and affected families are the erroneous belief that the disease cannot be prevented, treated or controlled; association of the disease with curses; and the belief that the disease runs in families through hereditary factors that are inevitable.

\section{Prevention and Management}

Podoconiosis is unique in being an entirely preventable non-communicable disease. Primary prevention method consists of avoiding or minimizing exposure to irritant soils by wearing shoes or boots and by covering floor surfaces inside traditional huts, in endemic areas. Secondary prevention involves training in a simple lymphoedema treatment regime. The regime includes daily foot-washing with soap, water and antiseptic, use of a simple emollient, bandaging in selected patients, elevation of the leg, controlled exercises, and use of socks and shoes (WHO, 2011 and Davey et al. 2007).

Tertiary prevention encompasses secondary prevention measures, elevation and compression of the affected leg, and, in selected cases, removal of prominent nodules. More radical surgery is no longer recommended since patients unable to scrupulously avoid contact with soil experience recurrent swelling which is more painful than the original disease because of scarring. Social rehabilitation is vital, and includes training treated patients in skills that enable them to generate income without contact with irritant soil (WHO, 2011)

There are a few podoconiosis control program efforts being undertaken in health centers and health posts in Ethiopia (FMOH, 2013). However, the success stories of the past ten years in Ethiopia implied that elimination of podoconiosis is achievable (Derib et al. 2013b).

Currently, there are 15 outreach clinics that have access to over 30,000 patients per year in Ethiopia. Preventive activities undergoing include health education at schools, kebeles, churches and mosques and at various social events; provision of locally produced shoes at subsidized prices or for free; and organization of 'Network Groups 'involving community members (FMOH, 2013and Molla et al. 2012).

Studies also indicated that, mapping of the disease burden of podoconiosis will help target resources, monitor control progress, and advocate for investment in podoconiosis prevention and control measures. After defining the target population and identifying the best approaches through research and in consultation with international experts, the goal of elimination can be fulfilled "within our lifetimes"(Derib et al. 2013b).

\section{Conclusion}

Low understanding and unfavorable attitudes towards podoconiosis contribute to severe stigmatization suffered by patients and their families. Lack of knowledge about the disease's risk factors, transmission, care and prevention is common among both the community and health professionals living in podoconiosis endemic areas. The economic effect of podoconiosis on patients and affected patients' families is also huge. There is a vicious cycle of poverty and podoconiosis. Poverty contributes to the disease because patients do not wear shoes is inability to afford them and podoconiosis contributes to poverty because patients are half as productive as their age and sex-matched non-affected controls. Generally, patients are now facing two solid factors, discrimination and poverty.

\section{List of abbreviations}

QoL: Quality of life

FMOH: Federal ministry of health

NTDs: Neglected tropical diseases

SNNPR: Southern nations, nationalities, and peoples' region

USD: United States Dollar

WHO: World health organization

\section{References}

[1] Davey, G.(2008). Podoconiosis: let Ethiopia lead the way. Ethiop.J.HealthDev,22(1).

[2] Davey, G., E. Gebrehanna, et al. (2007). "Podoconiosis: a tropical model for gene-environment interactions?" Trans $R$ Soc Trop Med Hyg, 101(1): 91-96.

[3] Deribe, K., Brooker, SJ.,Pullan, RL., Hailu, A., Enquselassie, F., Reithinger, R. Newport, M. and Davey, G. (2013a). Spatial distribution of podoconiosis in relation to environmental factors in Ethiopia: a historical review. PLoS One, 8 (7). e68330. ISSN 1932-6203. 
[4] Deribe, K., Tomczyk, S. and Tekola-Ayele, F. (2013b). Ten Years of Podoconiosis Research in Ethiopia. PLoSNegl Trop Dis,7(10): e2301. doi:10.1371/journal.pntd.0002301.

[5] FMOH (2013). National Master Plan for Neglected Tropical Diseases (NTDs). Addis Ababa, Federal Ministry of Health of Ethiopia.

[6] Molla, Y., Tomczyk, S., Amberbir, T., Tamiru, A. and Davey, G.(2012). Patients' perceptions of podoconiosis causes, prevention and consequences in East and West Gojam, Northern Ethiopia.BMC Public Health, 12:828.

[7] Molla, Y., Wardrop, N., Blond, J., Baxter, P., Newport, M., Atkinson, P. and Davey, G.(2014). Modeling environmental factors correlated with podoconiosis: a geo-spatial study of non-filarial elephantiasis. Int J Health Geogr, 13:24.

[8] Mousleya, E., Deribe, K., Tamiru, A., and Davey, G.(2013). The impact of podoconiosis on quality of life in Northern Ethiopia. Health Qual Life Outcomes, 11:122.

[9] Mousleya, E., Deribe, K., Tamiru, A., Tomczyk, S., Hanlone, C. and Daveya, G. (2015). Mental distress and podoconiosis in Northern Ethiopia: a comparative cross-sectional study. Int Health 7: 16-25.
[10] Tekola, F., H. Mariam, D. and Davey, G.(2006). Economic costs of endemic non-filarial elephantiasis in Wolaita Zone, Ethiopia. Trop Med Int Health, 11 (7 ):1136-1144.

[11] TekolaAyele, F., A. Adeyemo, et al. (2012). "Using a "genomics tool" to develop disease prevention strategy in a low-income setting: lessons from the podoconiosis research project." J Community Genet.

[12] Tora, A.,Davey, G., and Tadele, G. (2011). Qualitative study on stigma and coping strategies of patients with podoconiosis in Wolaita zone, Southern Ethiopia. Int Health..3:176- 181.

[13] Tora, A., Franklin, H.,Deribe, K., Reda, A. and Davey, G.(2014). Extent of podoconiosis-related stigma in Wolaita Zone, Southern Ethiopia: a cross-sectional study.Springer Plus, 3:647.

[14] WHO, http://www.who.int/neglected_diseases/diseases/podoconiosis/ en/ neglected tropical diseas. WHO, Geneva. 